\title{
The Exact Vacuum Solution for Kasner Metric from Bianchi Type-I Cosmological Model
}

\author{
Ahmed M. Al-Haysah \\ Department of Mathematics \\ Faculty of Education and Sciences-Rada'a \\ Albaydha University, Albaydha, Yemen
}

\author{
A.H. Hasmani \\ Department of Mathematics \\ Sardar Patel University \\ Vallabh Vidyanagar-388120, Gujarat India
}

\begin{abstract}
An exact solution of the vacuum Einstein field equations (VEFEs) has been obtained of a spatially homogeneous and anisotropic (SHA) Bianchi type-I cosmological model by Kasner. The Kasner metric is shown to be a special case, and the exact vacuum solution of Kasner form model is obtained. Some physical properties of the model have been discussed.
\end{abstract}

AMS Subject Classification: 83D05, 83F05, 83C15.

\section{Keywords}

Bianchi type-I, Vacuum solution, Cosmological model, Kasner form

\section{INTRODUCTION}

The simplest models are the Bianchi type-I cosmologies in class $A$ with $n^{(i)}=0$, i.e. $C_{j k}^{i}=0$, where $n^{(i)}$ is the parameters various symmetry types with values $0, \pm 1$ and $C_{j k}^{i}$ are the structure constants of the Lie algebra of the homogeneity group. So that all three Killing vectors (the group generators) commute. They contain the standard Einstein de-sitter model with flat spatial hyper-surfaces (curvature index $k=0$ ). In the vacuum case, all Bianchi type-I models are given by the well-known 1-parameter family of Kasner metrics (found in 1921 by E. Kasner and in 1933 by G. Lemaitre without considering the Bianchi groups) [1]

The Kasner space-time is an exact solution of the VEE $R_{i j}=0$, and the solutions play an important role in the discussion of certain cosmological questions. In another way, the Kasner solutions are obtained if the energy-momentum tensor $T_{i j}$ vanishes and the isometry group on spatial slices is the trivial one. Deruelle and Sasaki 2003 [2] were considered the Kasner metrics in the GaussBonnet case.

The purpose of this paper is to obtain an exact solution of the vacuum FEs from a SHA Bianchi type-I cosmological model. The well-known Kasner solution is obtained as a particular case.

\section{BIANCHI TYPE-I COSMOLOGICAL MODEL}

Let us consider the simplest vacuum SHA Bianchi type-I cosmological solution. This is the so called Kasner solution. The metric of Bianchi type-I is given by [3]

$$
d s^{2}=d t^{2}-\sum_{i=1}^{3} A_{i}^{2}(t) d x_{i}^{2},
$$

where $A_{i}, i=1,2,3$ are functions of time $t$ which are called cosmic scale factors [3]. Note that if $A_{1}=A_{2}=A_{3}=a$ we encounter the Friedmann-Robertson-Walker (FRW) solution with $k=0$. The computations of the Ricci tensor $R_{i j}$ and its spur using Mathematica [4] and [5]; the non-vanishing components are,

$$
\begin{aligned}
& R_{11}=-\dot{H}_{1}-\theta H_{1}, \\
& R_{22}=-\dot{H}_{2}-\theta H_{2}, \\
& R_{33}=-\dot{H}_{3}-\theta H_{3}, \\
& R_{44}=\dot{\theta}+H_{1}^{2}+H_{2}^{2}+H_{3}^{2},
\end{aligned}
$$

where an overhead dot denotes derivative with respect to time $t$ and $H_{1}, H_{2}, H_{3}$ and $\theta$ are the directional Hubble parameters (HPs) in the direction of $x_{1}, x_{2}, x_{3}$ and scalar expansion respectively gives

$$
H_{1}=\frac{\dot{A_{1}}}{A_{1}}, H_{2}=\frac{\dot{A_{2}}}{A_{2}}, H_{3}=\frac{\dot{A_{3}}}{A_{3}} \text {, and } \theta=H_{1}+H_{2}+H_{3} \text {. }
$$

The energy -momentum conservation condition is of the following form

$$
\dot{\rho}+\theta(\rho+p)=0,
$$

where $\rho$ is the proper energy density and $p$ is the isotropic pressure Because it's consider the vacuum solution, $T_{i j}=0$.

The EFEs are given by

$$
R_{i j}-\frac{1}{2} R g_{i j}=0,
$$

where the corresponding Ricci scalar $R$ is given by

$$
R=2\left[H_{1} H_{2}+H_{2} H_{3}+H_{1} H_{3}+\dot{\theta}+\theta^{2}\right] .
$$

The "44" component of the Einstein field Equations (8) for the metric (1) lead to

$$
R_{44}-\frac{1}{2} R=-\left[H_{1} H_{2}+H_{2} H_{3}+H_{1} H_{3}\right]=0 .
$$

As the result at Equation [10, from the definition of $\theta$ it's found that

$$
\theta^{2}=\left(H_{1}+H_{2}+H_{3}\right)^{2}=H_{1}^{2}+H_{2}^{2}+H_{3}^{2} .
$$




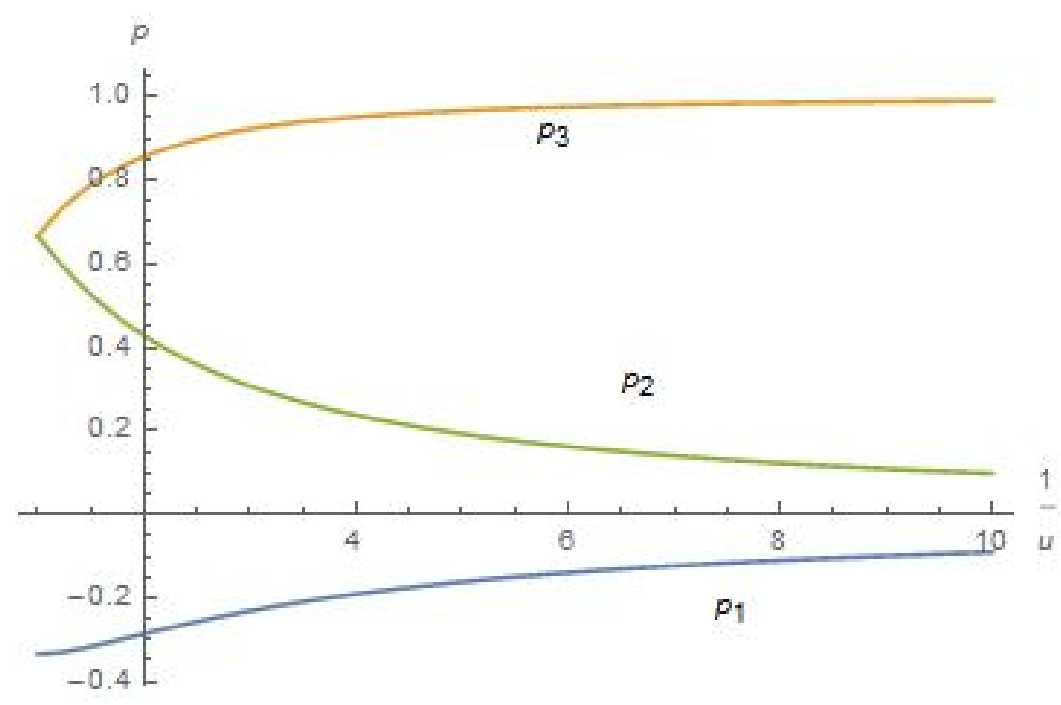

Fig. 1. The plot of Kasner indices $p_{i}, i=1,2,3$ versus parameter $\frac{1}{u}$.

Hence, using Equation (11) in Equation (5), we get

$$
R_{44}=\dot{\theta}+\theta^{2}=0 .
$$

If $\theta=0$, Equation 11 implies that $H_{1}=H_{2}=H_{3}=0$, i.e., $A_{1}$, $A_{2}$ and $A_{3}$ are constants. This gives Minkowski space-time.

If $\theta \neq 0$ the differential Equation (12) can be solved by separation of variables,

$$
\theta=\frac{1}{t-t_{0}}
$$

By a choice of the origin it can be set $t_{0}=0$. Then

$$
R_{11}=-\dot{H}_{1}-\theta H_{1}=0,
$$

Solving Equation (14), we get

$$
H_{1}=\frac{p_{1}}{t},
$$

for some constant $p_{1}$. Similarly by using Equations (3) and (4), we obtain respectively

$$
H_{2}=\frac{p_{2}}{t}, \text { and } H_{3}=\frac{p_{3}}{t},
$$

for some constants $p_{2}$ and $p_{3}$. So the Equations (11), (13) and (16) with (6) imply that $\theta=\frac{1}{t}=H_{1}+H_{2}+H_{3}=\frac{p_{1}}{t}+\frac{p_{2}}{t}+\frac{p_{3}}{t}$, we get

$$
\sum_{i=1}^{3} p_{i}=\sum_{i=1}^{3} p_{i}^{2}=1 .
$$

These are known as the Kasner relations. Now from $H_{1}=\frac{\dot{A_{1}}}{A_{1}}=$ $\frac{p_{1}}{t}$ we obtain that $A_{1}=A_{0} t_{1}^{p}$ and similarly for $A_{2}$ and $A_{3}$. Thus, the Kasner's metric can therefore be written as

$$
d s^{2}=d t^{2}-\sum_{i=1}^{3} t^{2 p_{i}} d x_{i}^{2},
$$

after the appropriate rescalings of $x_{i}, i=1,2,3$. Here $p_{i}, i=$ $1,2,3$ are subject to Equation (17).

The Kasner metric (18) is a solution to the VEFEs, thus Ricci tensor $R_{i j}$, Ricci scalar $R$ and its spur identify vanishes for any choice of exponents satisfying the Kasner conditions. The full Riemann tensor vanishes only when a single $p_{i}=1, i=1,2,3$ and the rest vanish, in which case the space is flat.

For $t>0$ the Kasner metric metric (18) describes an expanding homogeneous anisotropic universe, while for $t<0$ the contracting universe.

The exponents $p_{i}, i=1,2,3$ which is called the Belinskii, Khalatnikov, and Lifshitz (BKL) in the 1960's and 70's [6 7 8] parametrization can be parametrized by a real variable $u \geq 1$. Suppose without loss of generality that $p_{1}<p_{2}<p_{3}$, then

$$
\begin{aligned}
& p_{1}(u)=\frac{-u}{1+u+u^{2}}, \\
& p_{2}(u)=\frac{1+u}{1+u+u^{2}}, \\
& p_{3}(u)=\frac{u(1+u)}{1+u+u^{2}},
\end{aligned}
$$

as the parameter $u$ varies in the range ( see Figure 3)

$$
1 \leq u<+\infty \text {. }
$$

Figure 1 is a plot of $p_{i}, i=1,2,3$ versus parameter $\frac{1}{u}$. The numbers $p_{1}(u)$ and $p_{3}(u)$ are monotonously increasing while $p_{2}(u)$ is monotonously decreasing function of the parameter $u$.

The parameterization for $u<1$ leads to the same range by following the inversion property

$$
\begin{aligned}
& p_{1}\left(\frac{1}{u}\right)=p_{1}(u), \\
& p_{2}\left(\frac{1}{u}\right)=p_{3}(u),
\end{aligned}
$$




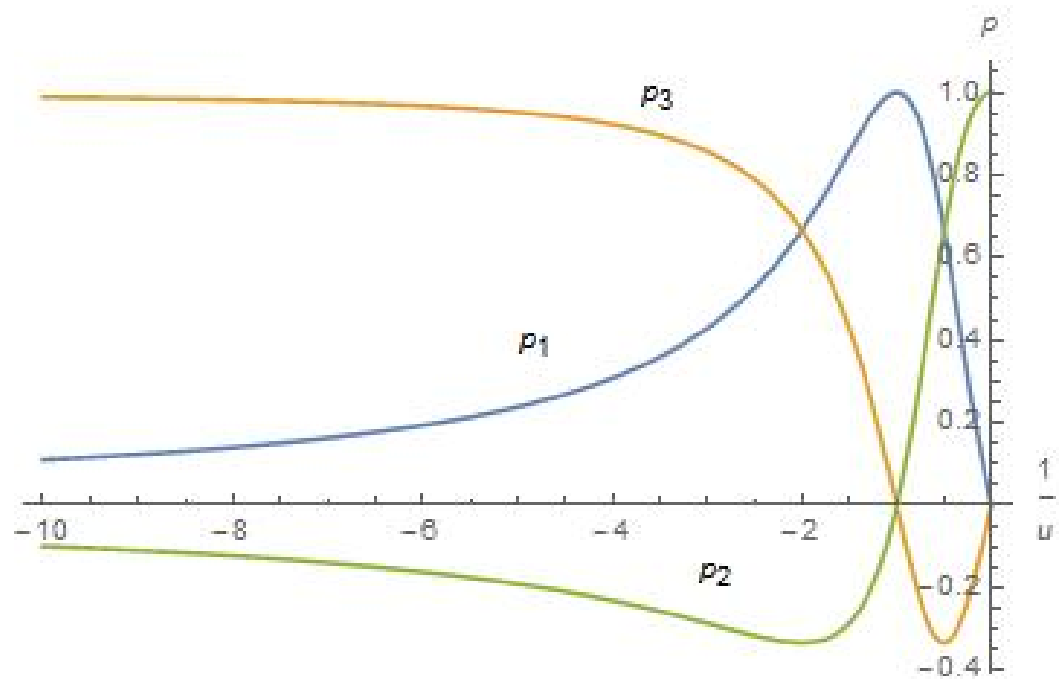

Fig. 2. The plot of Kasner indices $p_{i}, i=1,2,3$ versus parameter $\frac{1}{u}$.

$$
p_{3}\left(\frac{1}{u}\right)=p_{2}(u) .
$$

Figure 2 is a plot of $p_{i}, i=1,2,3$ versus parameter $\frac{1}{u}$. The numbers $p_{1}(u), p_{2}(u)$ and $p_{3}(u)$ are monotonously increasing and decreasing function of the parameter $u$.

The quantity $p_{1} p_{2} p_{3}$ is typically replaced by the Kasner parameter $u$ through

$$
p_{1} p_{2} p_{3}=\frac{-u^{2}(1+u)^{2}}{\left(1+u+u^{2}\right)^{3}}, \quad u \in[1, \infty) .
$$

Looking for explicit values of those exponents one find that except for the solutions with one index $p_{i}=1, i=1,2,3$ the others vanish, which could be proved in correspondence with the Minkowsky space solution (MSS) the Kasner indexes must be distributed in the following way

$$
\begin{gathered}
\frac{-1}{3} \leq p_{1} \leq 0, \\
0 \leq p_{2} \leq \frac{2}{3}, \\
\frac{2}{3} \leq p_{3} \leq 1 .
\end{gathered}
$$

The Equations (19, 20) and 21) imply that except for the two sets of Kasner exponents $(1,0,0)$ or $(2,-1,-1)$ and $\left(\frac{-1}{3}, \frac{2}{3}, \frac{2}{3}\right)$, all other sets of exponents contain one negative number and two positive numbers which are all different. The Kasner metric belongs to the homogeneous Bianchi type-I classification of the metric.

We come back to the spatial form of the Kasner metric (17) and (18), one sees that the requirement of symmetry in the plane between the $x_{2}$ and $x_{3}$ directions implies the condition

$$
p_{2}=p_{3} .
$$

Figure 3 is a plot of $p_{i}, i=1,2,3$ versus parameter $\frac{1}{u}$. The domain of $u$ is $[1, \infty)$; for lower values of $u$ the inversion prop- erty 23, 24 and 25 holds. The numbers $p_{1}(u)$ and $p_{3}(u)$ are monotonously increasing while $p_{2}(u)$ is monotonously decreasing function of the parameter $u$.

It is easy to see that there are two solutions of Equation (17) which satisfy the condition 30 .

\subsection{Flat space-time}

The flat space-time should be noted that with

$$
p_{1}=1, p_{2}=p_{3}=0 .
$$

In this case, the metric becomes

$$
d s^{2}=d t^{2}-t^{2} d x_{1}^{2}-\sum_{i=2}^{3} d x_{i}^{2} .
$$

which can be transformed to a metric of a flat space using a coordinate transformation. Due to this reason, the Kasner metric with any of the Kasner exponents unite and the others vanish is called the flat Kasner metric. The classical flat Kasner space-time has a non-curvature singularity at $t \rightarrow 0$.

This case was given by Taub (1951) [9], other rediscoveries were listed by Harvey (1990) [10] It is well-known that the Rindler space-time [11] represents a part of the Minkowski space-time rewritten in the coordinates connected with an accelerated observer by the transformation

$$
t \sinh x^{3}=\xi, \quad t \cosh x^{3}=\tau .
$$

It is worth noting that in this particular case. There is a coordinate singularity at $t \rightarrow 0$.

It's conclude that in the Bianchi type-I cosmology, which corresponds to a flat but anisotropic universe, there are three different scale factors referred to the spatial axes with two of them that increase with time and one which conversely decreases. 


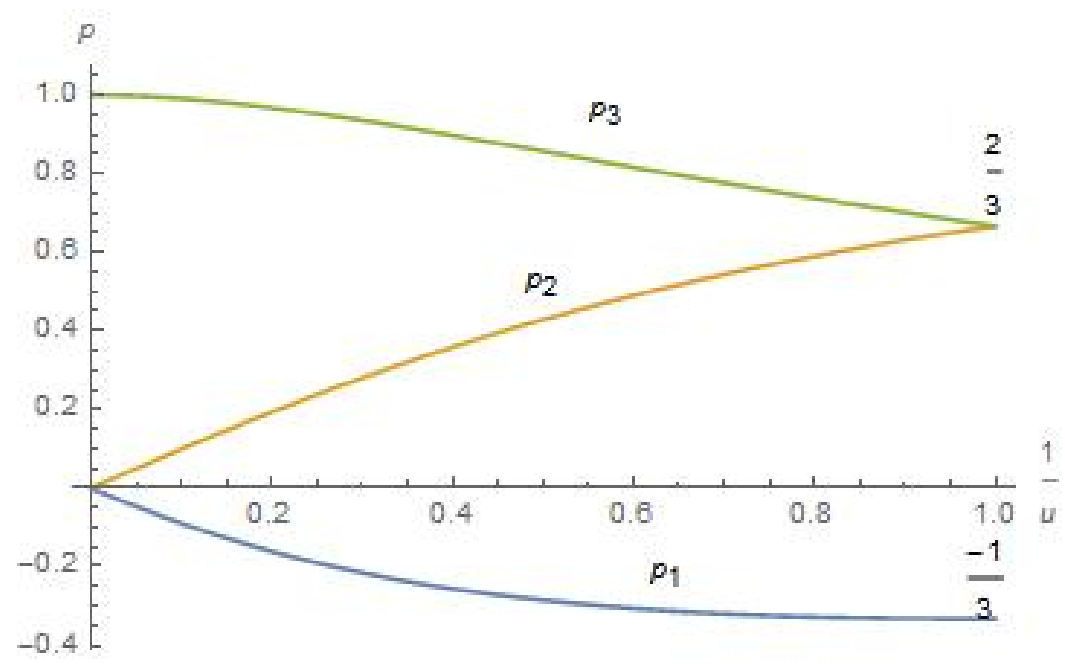

Fig. 3. The plot of Kasner indices $p_{i}, i=1,2,3$ versus parameter $\frac{1}{u}$.

\subsection{Non-flat space-time}

The non-flat plane symmetric should be noted that with

$$
p_{1}=\frac{-1}{3}, p_{2}=p_{3}=\frac{2}{3} .
$$

In this case, the metric becomes

$$
d s^{2}=d t^{2}-t^{\frac{-2}{3}} d x_{1}^{2}-t^{\frac{4}{3}} \sum_{i=2}^{3} d x_{i}^{2} .
$$

This particular solution was found by Weyl [12] and Levi-Civita [13] before the work of Kasner. This solution describes a universe, where a real curvature singularity is present at $t \rightarrow 0$.

\section{PHYSICAL AND GEOMETRICAL PROPERTIES OF THE KASNER MODEL}

The average scale-factor $a(t)$, and spatial volume $V$ are given by,

$$
V=\sqrt{-g}=a^{3}=t^{p_{1}+p_{2}+p_{3}}=t .
$$

Note that the spatial volume $V$ of a Kasner metric grows with time $t$ thus in the limit $t \rightarrow 0$ we have a Big-Bang (BB) like singularity and vanishes at $t=0$. It expands exponentially as $t$ increases and becomes infinitely large as $t \rightarrow \infty$.

The mean HPs, deceleration parameter (DP) and scalar expansion $\theta$ are

$$
\begin{aligned}
H & =\frac{\dot{a}}{a}=\frac{1}{3 t}, \\
q(t) & =\frac{-\ddot{a}}{a H^{2}}=2 \\
\theta & =3 H=\frac{1}{t} .
\end{aligned}
$$

Those do not depend on the supremacy of any of the axis. The directional HPs in the direction of $x_{1}, x_{2}$ and $x_{3}$ are obtained as

$$
H_{i}=\frac{p_{i}}{t}, i=1,2,3 \text { (no sum). }
$$

We observed that the HPs, directional HPs, and scalar expansion start with infinite value at $t=0$ and then become constant (decreasing function of time $t$ ) after some finite time $t$.

The shear scalar $\sigma^{2}$, and the average anisotropy parameter $\bar{A}$, which are defined as

$$
\begin{aligned}
& \sigma^{2}=\frac{1}{2}\left[\sum_{i=1}^{3} H_{i}^{2}-3 H^{2}\right]=0, \\
& \bar{A}=\frac{1}{3}\left[\sum_{i=1}^{3}\left(\frac{H_{i}-H}{H}\right)^{2}\right]=2 \neq 0 .
\end{aligned}
$$

The average anisotropy parameter $\bar{A} \neq 0$ is constant throughout the evolution of the universe, which implies that the Kasner model is anisotropic.

The shear parameter is given by

$$
\Sigma^{2}=\frac{\sigma^{2}}{3 H^{2}}=0
$$

The shear scalar $\sigma^{2}$ and shear parameter is zero throughout the evolution of the universe.

\section{CONCLUSION}

Exact vacuum solution of the Kasner metric from Bianchi TypeI models is obtained. The Kasner metric from Equation (18) describes an anisotropic space where volumes $V$ linearly grow with time, while linear distances grow along with two directions and decrease along the third one, different from the FRW solution where all distances contract towards the singularity with the same behavior. This metric has only one non-eliminable singularity in $t=0$ with the single exception of the case $p_{1}=1, p_{2}=p_{3}=0$ mentioned above, corresponding to the standard Euclidean space. 


\section{Acknowledgement}

The authors are thankful to the University Grant Commission, India for providing financial support under UGC-SAP-DRS(III) provided to the Department of Mathematics, Sardar Patel University, Vallabh Vidyanagar, where the work was carried out. AMA is also would like to thank the Al-Baydha University and Government of the Republic of Yemen for providing financial support. The authors are also thankful to the anonymous referee for critical comments that improved quality of the manuscript a lot.

\section{REFERENCES}

[1] Bicak, J. 1999. Selected solutions of Einsteins field equations: their role in general relativity and astrophysics. In Einsteins field equations and their physical implications. Springer, pp. $1-126$.

[2] Deruelle, N., and Sasaki, M. 2003. Newtons law on an Einstein gauss-bonnet brane. Progress of theoretical physics, Volume 110, No. 3, pp. 441-456.

[3] Hasmani, A. H., and Al-Haysah, A. M. 2019. Exact solutions for Bianchi type-I cosmological models in $f(R)$ theory of gravity. Applications and Applied Mathematics: An International Journal (AAM), Volume 14, No. 1, pp. 334-348.

[4] Hasmani, A. H. 2010. Algebraic computation of newmannpenrose scalars in general relativity using mathematica. Journal of Science, Volume 1, pp. 82-83.

[5] Hasmani, A. H., and Rathva, G. A. 2007. Algebric computations in general relativity using mathemetica. Prajna-J. of Pure and Applied Sciences, Volume 15, No. 111, pp. 77-81.

[6] Belinskii, V. A., Khalatnikov, I. M., and Lifshitz, E. M. 1970. Oscillatory approach to a singular point in the relativistic cosmology. Advances in Physics, Volume 19, No. 80, pp. 525573.

[7] Belinskii, V. A., Khalatnikov, I. M., and Lifshitz, E. M. 1982. A general solution of the Einstein equations with a time singularity. Advances in Physics, Volume 31, No. 6, pp. 639-667.

[8] Lifshitz, E. M., Khalatnikov, I. M., Beeby, J., et al. 1992. Investigations in relativistic cosmology. In Perspectives in Theoretical Physics. Elsevier, pp. 527-597.

[9] Taub, A. H. 1951. Empty space-times admitting a three parameter group of motions. Ann. Math, Volume 53, No.3, pp. 472-490.

[10] Harvey, A. 1990. Will the real kasner metric please stand up. General Relativity and Gravitation, Volume 22, No. 12, pp. 1433-1445.

[11] Kamenshchik, A. Y., and Vardanyan, T. 2019. Exact solutions of the Einstein equations for an infinite slab with a constant energy density. Physics Letters B, Volume 792, pp. 430-435.

[12] Weyl, H. 1917. Zur gravitationstheorie. Annalen der Physik, Volume 359, No. 18, pp. 117-145.

[13] Levi-Civita, T. 1918 Levi-civita rend. acc. lincei 27, 220; levicivita, t.(1918). Rend. Acc. Lincei, Volume 27, pp. 240-252.

[14] Giovanni, M., Valerio, B. M., and Riccardo, B. 2011. Primordial cosmology. World Scientific. 